\title{
Editorial: Best Practices on Advanced Condition Monitoring of Rail Infrastructure Systems, Volume II
}

\author{
Stefano Bruni ${ }^{1}$, Serdar Dindar ${ }^{2}$ and Sakdirat Kaewunruen ${ }^{3 *}$ \\ ${ }^{1}$ Department of Mechanical Engineering, Politecnico di Milano, Milan, Italy, ${ }^{2}$ Department of Civil Engineering, Izmir Kâtip Çelebi \\ University, Izmir, Turkey, ${ }^{3}$ School of Engineering, University of Birmingham, Birmingham, United Kingdom
}

Keywords: rail, railway, infrastructure, condition, monitoring, social impact

Editorial on the Research Topic

Best Practices on Advanced Condition Monitoring of Rail Infrastructure Systems, Volume II

None of us would have imagined that Covid-19 would last this long. However, recent insights demonstrate that we as a human will need to adapt and live with it for quite some time or even perhaps forever. Interestingly, due to COVID19, many news reports have shown a significant drop in public transport usages. In fact, the number of train passengers are at the lowest level in decades as demonstrated in Figure 1, revealing some daily facts of national rail usage in the United Kingdom over the period. The coronavirus causes severe long-lasting imprints on many sectors, and railway is not exempted. With the necessity to survive, railway sectors must adapt to enable and support the recovery of economic activities now.

Various standard and practice for managing infrastructure systems have been recently updated to tackle the challenges. However, with the risk of more and more staff getting contacts with the virus, contingency plan is necessary. Accordingly, there is an essential need to monitor and manage the integrity of the railway systems (including rolling stocks, infrastructures, signaling, and other aspects) through advanced condition monitoring across the networks. Such the essential need is the key theme in this special research topic that is emphasized on rail infrastructure systems designed to cope with multi hazards and extreme events. The second volume of this Research Topic will continue bringing together the research and innovation associated with rail infrastructure systems issues related to advanced condition monitoring, design and maintenance of infrastructure systems and components, and societal impacts. These aspects are the essential technological enablers to enhance social and economic connections where communities can quickly take up the opportunities offered by increased mobility (Kaewunruen et al., 2016). The technological enablers underpin 'The Sendai Framework for Disaster Risk Reduction' endorsed by United Nations Office for Disaster Risk Reduction. They will lead to: 1) promptly and effectively respond to any crisis; 2) assure the quality of everyday life; and 3) leave no one behind.

This research topic presents a set of new research findings stemming from the H2020 Marie Skłodowska-Curie Actions (MSCA)'s RISEN Project (Rail Infrastructure Systems Engineering Network). This project has received funding from the European Union's Horizon 2020 research and innovation programme under grant agreement No 691135 (www.risen2rail.eu). The collaborative researches are aligned with United Nation's Sustainable Development Goals (SDGs) as well as European Directive's Plan S for Open Science.

Along this line, Edwards et al. presented a design process based on structural reliability analysis (SRA) concepts whereby target values for reliability indices $(\beta)$ for new designs are obtained and compared with existing designs for further design optimization. The results showed the need for increased sleeper center bending capacity. Additionally, a reduction in rail seat bending capacity of approximately $40 \%$ is justified, reducing the size of the rail seat cross 


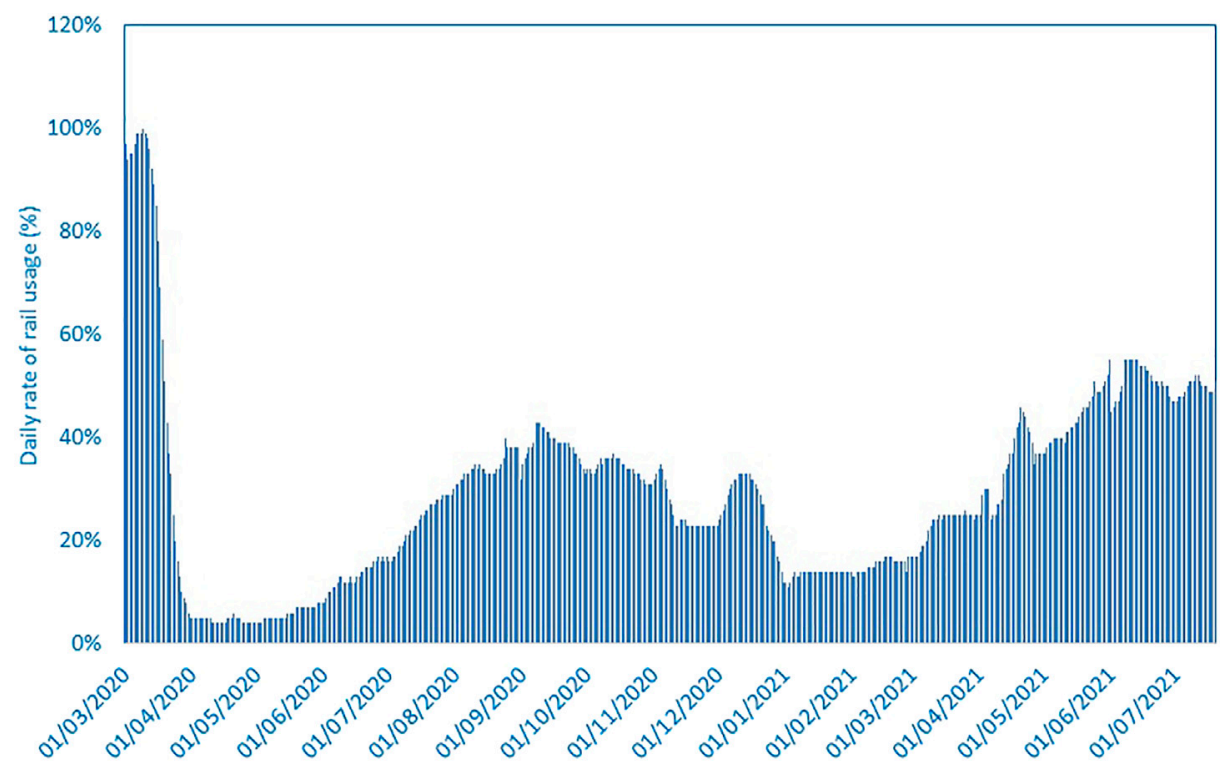

FIGURE 1 | Percentage of daily rate of national rail usage in the United Kingdom derived from the open data by United Kingdom Department for Transport (source: https://www.gov.uk/government/statistics/transport-use-during-the-coronavirus-covid-19-pandemic).

section by approximately the same magnitude. In most cases the proposed designs have fewer prestressing wires and a higher centroid of prestressing steel.

Kaewunruen et al. (2016) discussed about the design and testing procedures of composite materials, which have recently gained significant attention for applications in railway industry. In recent practice, composite sleepers and bearers have been used for bespoke replacements of aged timber components in critical areas such as switches and crossings, bridge transom sleepers, and special locations with either stiffness or clearance constraints. A new ISO standard has been drafted to accommodate the need to carry out standardized tests to benchmark the performance of polymeric composite sleepers and bearers. This study highlighted the test specifications in order to illustrate the profound insight into the test methods for polymeric composite sleepers in comparison with in situ conditions in real life situations. The study explored the effectiveness of the provision in the current design code for bending test methods under various support conditions. It vividly demonstrated that the test methods cannot fully represent in situ track conditions.

Pereira Silva et al. shared a new development of a ballast support condition back-calculator, a non-destructive instrumentation method and corresponding analysis tool that quantifies ballast pressure distributions under concrete sleepers without interrupting revenue service train operations. This study quantified the ballast pressure distributions beneath concrete sleepers under different types of rolling stock and evaluates how ballast support condition changes as a function of accumulated tonnage. It also demonstrated the potential of the back-calculator to provide a stand-alone non-invasive method to quantify ballast support conditions, sleeper health, and sleeper bearing stress. Back calculator data will aid the rail industry in optimizing tamping cycles, enhancing safety, and developing more representative concrete sleeper flexural designs based on actual support conditions.

Rungskunroch et al. highlighted the Paris Agreement, which has been launched as a global environmental policy. The agreement has been involved in the transportation industry, especially on railway and HSR networks. Implementing environmental concepts becomes a challenging issue for researchers and engineers in the railway industry. Despite the fact that railway sector is the lowest $\mathrm{CO} 2$ emitter, there have been several collaborations among railway industries, operators, policymakers, and other related sections to further respond to reduce global emission. The study provided three realistic key developments covering infrastructure, electric rail track, and vehicle's engine power. By following the proposed guidelines, the United Kingdom government's target to be net-zero carbon is more achievable.

Alawad and Kaewunruen discussed the emerging context of COVID-19 when the railway industry could employ new technology such as AI and related approaches (Internet of things (IoT), 5G, big data, and AI) for protecting and underpinning railway safety. Acknowledging already the solutions and potential measures that have been applied such as cleaning, disinfection, sanitization, redesign, physical and social distancing, relayout, ATP testing, or the air filtration and recycling air, the research highlighted the potential technology solutions in the industry for tackling COVID-19. The situation has led to rethinking how we consider and continue to benefit from technology in the current situation and for future crises, not only for the railway but for all businesses.

Liu et al. determined the casual effect and synergy of highspeed rail development on the modal transport changes in supply chain and logistics, which have not been considered well during 
the initial phase of any rail project design and development. This issue has impaired the systems integration and connectivity among the modes of transport in a region. This study provided the sensitivity analysis of supply chains via air-railroad freight transportation and logistics stemming from the High Speed 2 case by the rigorous assessments into the capacity, performance and environmental changes that may follow the project's implementation. The research proposes a new method for estimation of consequences from a new transport project construction. The research findings demonstrate slight beneficial changes in freight transportation and logistics with a high potential for development; and reveal the project's weaknesses

\section{REFERENCE}

Kaewunruen, S., Sussman, J. M., and Matsumoto, A. (2016). Grand Challenges in Transportation and Transit Systems. Front. Built Environ. 2, 4. doi:10.3389/ fbuil.2016.00004

Conflict of Interest: The authors declare that the research was conducted in the absence of any commercial or financial relationships that could be construed as a potential conflict of interest.

Publisher's Note: All claims expressed in this article are solely those of the authors and do not necessarily represent those of their affiliated and opportunities for better systems integration and business synergy.

These papers provide exciting new insights and state-of-theart knowledge for actions towards smarter and more resilient railway systems. The topic editors are grateful to the review editors and associated editors.

\section{AUTHOR CONTRIBUTIONS}

All authors listed have made a substantial, direct, and intellectual contribution to the work and approved it for publication.

organizations, or those of the publisher, the editors and the reviewers. Any product that may be evaluated in this article, or claim that may be made by its manufacturer, is not guaranteed or endorsed by the publisher.

Copyright (c) 2021 Bruni, Dindar and Kaewunruen. This is an open-access article distributed under the terms of the Creative Commons Attribution License (CC $B Y)$. The use, distribution or reproduction in other forums is permitted, provided the original author(s) and the copyright owner(s) are credited and that the original publication in this journal is cited, in accordance with accepted academic practice. No use, distribution or reproduction is permitted which does not comply with these terms. 